Azeddine Aissaoui Fqayeh, Abdelmoujib Benkirane and Mostafa El Moumni (Fez)

\title{
ENTROPY SOLUTIONS FOR NONLINEAR UNILATERAL PARABOLIC INEQUALITIES IN ORLICZ-SOBOLEV SPACES
}

Abstract. We discuss the existence of entropy solution for the strongly nonlinear unilateral parabolic inequalities associated to the nonlinear parabolic equations $\frac{\partial u}{\partial t}-\operatorname{div}(a(x, t, u, \nabla u)+\Phi(u))+g(u) M(|\nabla u|)=\mu$ in $Q$, in the framework of Orlicz-Sobolev spaces without any restriction on the $N$-function of the Orlicz spaces, where $-\operatorname{div}(a(x, t, u, \nabla u))$ is a Leray-Lions operator and $\Phi \in C^{0}\left(\mathbb{R}, \mathbb{R}^{N}\right)$. The function $g(u) M(|\nabla u|)$ is a nonlinear lower order term with natural growth with respect to $|\nabla u|$, without satisfying the sign condition, and the datum $\mu$ belongs to $L^{1}(Q)$ or $L^{1}(Q)+W^{-1, x} E_{\bar{M}}(Q)$.

1. Introduction. Let $Q$ be the cylinder $\Omega \times(0, T)$, where $T>0$ and $\Omega$ is a bounded domain of $\mathbb{R}^{N}$ with the segment property, and let $M$ and $P$ be two $N$-functions such that $P \ll M$. In the present paper, we consider the following boundary value problem:

$$
\begin{cases}u \geq \psi \quad \text { a.e. in } Q, \\ \frac{\partial u}{\partial t}-\operatorname{div}(a(x, t, u, \nabla u)+\Phi(u))+g(u) M(|\nabla u|)=f \quad \text { in } Q \\ u=0 \quad \text { on } \partial \Omega \times(0, T) \\ u(x, 0)=u_{0}(x) & \text { in } \Omega,\end{cases}
$$

where $a: \Omega \times(0, T) \times \mathbb{R} \times \mathbb{R}^{N} \rightarrow \mathbb{R}^{N}$ is a Carathéodory function (that is, measurable with respect to $x$ in $\Omega$ for every $(t, s, \xi)$ in $\mathbb{R} \times \mathbb{R} \times \mathbb{R}^{N}$, and continuous with respect to $(s, \xi)$ in $\mathbb{R} \times \mathbb{R}^{N}$ for almost every $x$ in $\Omega$ ) such that for all $\xi$ and $\xi^{*}$ in $\mathbb{R}^{N}, \xi \neq \xi^{*}$,

2010 Mathematics Subject Classification: Primary 46E30; Secondary 35K85.

Key words and phrases: Orlicz-Sobolev spaces, nonlinear parabolic unilateral problems and nonlinear parabolic variational inequalities, $L^{1}$-data. 


$$
\begin{gathered}
a(x, t, s, \xi) \xi \geq \alpha M(|\xi|), \\
{\left[a(x, t, s, \xi)-a\left(x, t, s, \xi^{*}\right)\right]\left[\xi-\xi^{*}\right]>0,} \\
|a(x, t, s, \xi)| \leq c(x, t)+k_{1} \bar{P}^{-1} M\left(k_{2}|s|\right)+k_{3} \bar{M}^{-1} M\left(k_{4}|\xi|\right),
\end{gathered}
$$

where $c(\cdot, \cdot)$ belongs to $E_{\bar{M}}(Q), c \geq 0, k_{i} \geq 0(i=1,2,3,4)$ and $\alpha>0$. Moreover,

$$
\begin{aligned}
& \Phi: \mathbb{R} \rightarrow \mathbb{R}^{N} \text { is a continuous function, } \\
& f \in L^{1}(Q), \\
& u_{0} \in L^{1}(\Omega), \quad u_{0} \geq \psi(x) \text { a.e. in } \Omega, \\
& \psi \in L^{\infty}(\Omega) \cap W_{0}^{1, x} E_{M}(\Omega), \quad \psi(x) \leq 0 \text { a.e. in } \Omega, \\
& g: \mathbb{R}^{+} \rightarrow \mathbb{R}^{+} \text {is an integrable continuous function. }
\end{aligned}
$$

REMARK 1.1. We remark that if $u_{0} \in L^{1}(\Omega), u_{0} \geq 0$, then the convex subset $\left\{v \in L^{1}(\Omega): v \geq \psi\right\}$ is nonempty.

In $[\mathrm{P}]$ where $\Phi \equiv 0$, the author has shown the existence of a renormalized solution for the corresponding equation. In $[\mathrm{P}$, the function $a(x, t, u, \nabla u)$ was assumed to satisfy a polynomial growth condition with respect to $u$ and $\nabla u$. When trying to relax this restriction on the function $a(x, t, u, \nabla u)$, we are led to replace the space $L^{p}\left(0, T ; W_{0}^{1, p}(\Omega)\right)$ by an inhomogeneous Orlicz-Sobolev space $W^{1, x} L_{M}(Q)$ built from an Orlicz space $L_{M}$ instead of $L^{p}$, where the $N$-function $M$ which defines $L_{M}$ is related to the actual growth of the Carathéodory function. Recently M. Kbiri Alaoui et al. [KMS proved the existence result for the obstacle problem associated to (1.1) in the setting of Orlicz-Sobolev spaces where $\Phi \equiv 0$. The above problem does not admit, in general, a weak solution since the fields $a(x, t, u, \nabla u)$ and $\Phi(u)$ do not belong in $\left(L_{\text {loc }}^{1}(Q)\right)^{N}$ in general. For analogous elliptic or parabolic problems in the setting of Sobolev spaces or Orlicz-Sobolev spaces, we refer the reader to $\mathrm{ABT}, \mathrm{ABM} 15, \mathrm{ABM}, \mathrm{ABMR}, \mathrm{AB}, \mathrm{BE}, \mathrm{D}, \mathrm{E}, \mathrm{EM}$, EM3, GM, L, R, YBM1, YBM2.

This paper is motivated by recent advances in mathematical modeling of non-Newtonian fluids and elastic mechanics, in particular, electrorheological fluids (smart fluids). This important class of fluids is characterized by the change of viscosity which depends on the electric field. These fluids, also known as ER fluids, have many applications in elastic mechanics, fluid dynamics etc.

The scope of the present paper is to solve the obstacle problem associated to (1.1) in the case where $f \in L^{1}(Q)+W^{-1, x} E_{\bar{M}}(Q)$ and without assuming any growth restriction on $M, \Phi(u) \not \equiv 0$, while the function $g(u) M(|\nabla u|)$ does not satisfy the sign condition. The existence of solutions is proved via a sequence of penalized problems. 
2. Existence results. This section is devoted to establishing the following existence theorem.

Theorem 2.1. Assume that $1.2-(1.8)$ hold. Then there exists a solution of problem (1.1) in the following sense:

$$
\left\{\begin{array}{c}
u \geq \psi \quad \text { a.e. in } Q, \quad T_{k}(u) \in W_{0}^{1, x} L_{M}(Q), \quad S_{k}(u(\cdot, t)) \in L^{1}(\Omega), \\
\int_{\Omega} S_{k}(u(T)-v(T)) d x+\left\langle\frac{\partial v}{\partial t}, T_{k}(u-v)\right\rangle+\int_{Q} a(x, t, u, \nabla u) \nabla T_{k}(u-v) d x d t \\
+\int_{Q} \Phi(u) \nabla T_{k}(u-v) d x d t \leq \int_{Q} g(u) M(|\nabla u|) T_{k}(u-v) d x d t \\
\quad+\int_{Q} f T_{k}(u-v) d x d t+\int_{\Omega} S_{k}\left(u_{0}-v(0)\right) d x
\end{array}\right.
$$

for all $k>0$ and $v \in W_{0}^{1, x} L_{M}(Q) \cap L^{\infty}(Q)$ such that $\partial v / \partial t \in W^{-1, x} L_{\bar{M}}(Q)$ $+L^{1}(Q)$ and $v \geq \psi$. Here $S_{k}$ is the truncation defined by $S_{k}(\tau)=\int_{0}^{\tau} T_{k}(s) d s$ where the standard truncation function $T_{k}, k>0$, is defined for all $s \in \mathbb{R}$ by $T_{k}(s)=\max \{-k, \min \{k, s\}\}$.

The proof is divided into four steps.

SteP 1: Approximate problems and a priori estimate. Consider the following approximate problem:

$$
\left\{\begin{array}{l}
\frac{\partial u_{n}}{\partial t}-\operatorname{div}\left(a\left(x, t, u_{n}, \nabla u_{n}\right)+\Phi_{n}\left(u_{n}\right)\right)-n T_{n}\left(\left(u_{n}-\psi\right)^{-}\right) \\
\quad=g\left(u_{n}\right) M\left(\left|\nabla u_{n}\right|\right)+f_{n} \quad \text { in } Q \\
u_{n}(x, 0)=u_{0 n}(x) \text { in } \Omega
\end{array}\right.
$$

where we have set $\Phi_{n}(s)=\Phi\left(T_{n}(s)\right)$. For fixed $n>0$, since $\Phi$ is continuous, it is obvious that $\left|\Phi_{n}(t)\right| \leq \max _{|t| \leq n}|\Phi(t)|=C_{n}$. Moreover, the sequence $\left(f_{n}\right) \subset \mathcal{D}(Q)$ is such that $f_{n} \rightarrow f$ strongly in $L^{1}(Q)$ and $\left(u_{0 n}\right) \subset \mathcal{D}(\Omega)$ is such that $u_{0 n} \rightarrow u_{0}$ strongly in $L^{1}(\Omega)$. By Lemma 3.1 of KMS, there exists a weak solution $u_{n} \geq 0$ in $W_{0}^{1, x} L_{M}(Q)$ of problem 2.1). Let $h>0$ and consider the test function $v=T_{h}\left(u_{n}\right) \exp \left(\int_{0}^{u_{n}} g(s) d s\right)$ in 2.1 . We have

$$
\begin{aligned}
& \left\langle\frac{\partial u_{n}}{\partial t}, T_{h}\left(u_{n}\right) \exp \left(\int_{0}^{u_{n}} g(s) d s\right)\right\rangle \\
& \quad+\int_{\left\{u_{n} \leq h\right\}} a\left(\cdot, u_{n}, \nabla u_{n}\right) \nabla u_{n} \exp \left(\int_{0}^{u_{n}} g(s) d s\right) d x d t \\
& \quad+\int_{Q} a\left(\cdot, u_{n}, \nabla u_{n}\right) \nabla u_{n} T_{h}\left(u_{n}\right) g\left(u_{n}\right) \exp \left(\int_{0}^{u_{n}} g(s) d s\right) d x d t
\end{aligned}
$$




$$
\begin{aligned}
& +\int_{Q} \Phi_{n}\left(u_{n}\right) \nabla\left(T_{h}\left(u_{n}\right) \exp \left(\int_{0}^{u_{n}} g(s) d s\right)\right) d x d t \\
& -\int_{Q} n T_{n}\left(\left(u_{n}-\psi\right)^{-}\right) T_{h}\left(u_{n}\right) \exp \left(\int_{0}^{u_{n}} g(s) d s\right) d x d t \\
& =\int_{Q} g\left(u_{n}\right) M\left(\left|\nabla u_{n}\right|\right) T_{h}\left(u_{n}\right) \exp \left(\int_{0}^{u_{n}} g(s) d s\right) d x d t \\
& +\int_{Q} f_{n} T_{h}\left(u_{n}\right) \exp \left(\int_{0}^{u_{n}} g(s) d s\right) d x d t .
\end{aligned}
$$

The Lipschitz character of $\Phi_{n}$ and the Stokes formula together with the boundary condition $u_{n}=0$ on $(0, T) \times \partial \Omega$ make it possible to obtain

$$
\int_{Q} \Phi_{n}\left(u_{n}\right) \nabla\left(T_{h}\left(u_{n}-T_{k}\left(u_{n}\right)\right) \exp \left(\int_{0}^{u_{n}} g(s) d s\right)\right) d x d t=0 .
$$

Using (2.2) and (1.2), and since $u_{n} \geq 0$ gives $T_{h}\left(u_{n}\right) \geq 0$, we have

$$
\begin{aligned}
\left\langle\frac{\partial u_{n}}{\partial t}, T_{h}\left(u_{n}\right) \exp \left(\int_{0}^{u_{n}} g(s) d s\right)\right\rangle & \\
& +\int_{\left\{u_{n} \leq h\right\}} M\left(\left|\nabla u_{n}\right|\right) \exp \left(\int_{0}^{u_{n}} g(s) d s\right) \\
& -n \int_{Q} T_{n}\left(\left(u_{n}-\psi\right)^{-}\right) T_{h}\left(u_{n}\right) \exp \left(\int_{0}^{u_{n}} g(s) d s\right) d x d t \\
\leq & \int_{Q} f_{n} T_{h}\left(u_{n}-T_{k}\left(u_{n}\right)\right) \exp \left(\int_{0}^{u_{n}} g(s) d s\right) d x d t .
\end{aligned}
$$

We have

$$
\begin{aligned}
& \left\langle\frac{\partial u_{n}}{\partial t}, T_{h}\left(u_{n}\right) \exp \left(\int_{0}^{u_{n}} g(s) d s\right)\right\rangle \\
& \quad=\int_{\Omega} \int_{0}^{u_{n}(x, T)} T_{h}(s) \exp \left(\int_{0}^{s} g(s) d s\right)-\iint_{\Omega}^{u_{0}} T_{h}(s) \exp \left(\int_{0}^{s} g(s) d s\right) \geq-C h,
\end{aligned}
$$

where

$$
C=\left\|u_{0}\right\|_{L^{1}(\Omega)} \int_{0}^{u_{0}} T_{h}(s) \exp \left(\int_{0}^{u_{0}} g(s) d s\right)
$$

So, we obtain

$$
-n \int_{Q} T_{n}\left(\left(u_{n}-\psi\right)^{-}\right) T_{h}\left(u_{n}\right) \leq C h
$$


and since $\psi(x) \leq 0$ a.e. in $\Omega$,

$$
T_{h}\left(u_{n}\right) \leq T_{h}\left(\left(u_{n}-\psi\right)\right.
$$

and

$$
-\int_{Q} n T_{n}\left(\left(u_{n}-\psi\right)^{-}\right) T_{h}\left(u_{n}-\psi\right) \leq-\int_{Q} n T_{n}\left(\left(u_{n}-\psi\right)^{-}\right) T_{h}\left(u_{n}\right) \leq C h .
$$

Then

$$
\int_{Q} n T_{n}\left(\left(u_{n}-\psi\right)^{-}\right) \frac{T_{h}\left(\left(u_{n}-\psi\right)^{-}\right)}{h} \leq C .
$$

Letting $h$ to tend to zero, one has

$$
0 \leq \int_{Q} n T_{n}\left(\left(u_{n}-\psi\right)^{-}\right) \leq C .
$$

If we use $v=T_{k}\left(u_{n}\right) \exp \left(\int_{0}^{u_{n}} g(s) d s\right)$ as a test function in 2.1), then as above we obtain

$$
\int_{Q} M\left(\left|\nabla T_{k}\left(u_{n}\right)\right|\right) \exp \left(\int_{0}^{u_{n}} g(s) d s\right) \leq C_{1} k .
$$

Thus $\left(T_{k}\left(u_{n}\right)\right)_{n}$ is bounded in $W_{0}^{1, x} L_{M}(Q)$, and so there exist some $w_{k} \in$ $W_{0}^{1, x} L_{M}(Q)$ such that

$$
\begin{array}{ll}
T_{k}\left(u_{n}\right) \rightarrow w_{k} & \text { weakly in } W_{0}^{1, x} L_{M}(Q) \text { for } \sigma\left(\prod L_{M}, \prod E_{\bar{M}}\right), \\
T_{k}\left(u_{n}\right) \rightarrow w_{k} & \text { strongly in } E_{M}(Q) \text { and a.e. in } Q,
\end{array}
$$

where $\prod L_{M}$ is the product of $N+1$ copies of $L_{M}$ and $\prod E_{M}$ is the product of $N+1$ copies of $E_{M}$.

Let $\eta_{k}$ be the nondecreasing $C^{2}(\mathbb{R})$ function with

$$
\eta_{k}(s)= \begin{cases}s, & |s| \leq k / 2, \\ k \operatorname{sign}(s), & |s| \geq k .\end{cases}
$$

Multiplying the approximating equation by $\eta_{k}^{\prime}\left(u_{n}\right)$, we get

$$
\begin{array}{r}
\frac{\partial \eta_{k}\left(u_{n}\right)}{\partial t}-\operatorname{div}\left(a\left(x, t, u_{n}, \nabla u_{n}\right) \eta_{k}^{\prime}\left(u_{n}\right)\right)+a\left(x, t, u_{n}, \nabla u_{n}\right) \cdot \nabla u_{n} \eta_{k}^{\prime \prime}\left(u_{n}\right) \\
-\operatorname{div}\left(\Phi_{n}\left(u_{n}\right) \eta_{k}^{\prime}\left(u_{n}\right)\right)+\Phi_{n}\left(u_{n}\right) \eta_{k}^{\prime \prime}\left(u_{n}\right) \nabla u_{n} \\
=g\left(u_{n}\right) M\left(\left|\nabla u_{n}\right|\right) \eta_{k}^{\prime}\left(u_{n}\right)+f_{n} \eta_{k}^{\prime}\left(u_{n}\right)+n T_{n}\left(\left(u_{n}-\psi\right)^{-}\right) \eta_{k}^{\prime}\left(u_{n}\right)
\end{array}
$$

in the distribution sense. We deduce that $\eta_{k}\left(u_{n}\right)$ is bounded in $W_{0}^{1, x} L_{M}(Q)$ and $\partial \eta_{k}\left(u_{n}\right) / \partial t$ in $W^{-1, x} L_{\bar{M}}(Q)+L^{1}(Q)$. By the Corollary of [EM1], $\eta_{k}\left(u_{n}\right)$ is compact in $L^{1}(Q)$. 
In the same way as in $[\mathrm{P}]$ we obtain, for every $k>0$,

$$
\begin{array}{ll}
T_{k}\left(u_{n}\right) \rightarrow T_{k}(u) \quad \text { weakly in } W_{0}^{1, x} L_{M}(Q) \text { for } \sigma\left(\prod L_{M}, \prod E_{\bar{M}}\right), \\
T_{k}\left(u_{n}\right) \rightarrow T_{k}(u) \quad \text { strongly in } L^{1}(Q) \text { and a.e. in } Q .
\end{array}
$$

Now using the estimate 2.3 and Fatou's Lemma, we obtain

$$
(u-\psi)^{-}=0,
$$

and so $u \geq \psi$.

STEP 2: Almost everywhere convergence of the gradients

LEMMA 2.2. Let $u_{n}$ be a solution of the approximate problem (2.1). Then there exists a subsequence also denoted by $u_{n}$ such that

$$
\nabla u_{n} \rightarrow \nabla u \quad \text { a.e. in } Q \text {. }
$$

The proof is similar to Step 2 in [KMS. Now, there exists a subsequence also denoted by $u_{n}$ such that

$$
\nabla u_{n} \rightarrow \nabla u \quad \text { a.e. in } Q .
$$

We deduce that

$$
\begin{aligned}
a\left(\cdot, T_{k}\left(u_{n}\right), \nabla T_{k}\left(u_{n}\right)\right) \rightarrow a\left(\cdot, T_{k}(u),\right. & \left.\nabla T_{k}(u)\right) \\
& \text { in }\left(L_{\bar{M}}(Q)\right)^{N} \text { for } \sigma\left(\Pi L_{M}, \Pi E_{\bar{M}}\right) .
\end{aligned}
$$

STEP 3: Modular convergence of the truncations. We use the same technique as in [GM] in the parabolic case. The functions $v_{j}, \chi_{j}^{s}, \varepsilon(n, j, \mu, i, s, m)$, $\chi^{s}$ and $\varepsilon(n, j, s)$ below are as in Step 2 in [KMS. By using the same argument as in Step 2 in [KMS], we prove that

$$
\begin{array}{r}
\int_{Q}\left(a\left(\cdot, T_{k}\left(u_{n}\right), \nabla T_{k}\left(u_{n}\right)\right)-a\left(\cdot, T_{k}\left(u_{n}\right), \nabla T_{k}\left(v_{j}\right) \chi_{j}^{s}\right)\right)\left(\nabla T_{k}^{*}\left(u_{n}\right)-\nabla T_{k}\left(v_{j}\right) \chi_{j}^{s}\right) \\
\times \exp \left(\int_{0}^{u_{n}} g(s) d s\right) d x d t \leq \varepsilon(n, j, \mu, i, s, m),
\end{array}
$$

where

$$
T_{k}^{*}(s)=\left(\int_{0}^{T_{k}(s)} \exp \left(\int_{0}^{t} g(s) d s\right) d t\right)\left(\exp \left(-\int_{0}^{\infty} g(s) d s\right)\right) .
$$

We can also deduce that

$$
\begin{aligned}
\int_{Q}\left(a\left(\cdot, T_{k}\left(u_{n}\right), \nabla T_{k}\left(u_{n}\right)\right)-a\left(\cdot, T_{k}\left(u_{n}\right), \nabla T_{k}^{*}(u) \chi^{s}\right)\right)\left(\nabla T_{k}^{*}\left(u_{n}\right)-\nabla T_{k}^{*}(u) \chi^{s}\right) \\
\\
\times \exp \left(\int_{0}^{u_{n}} g(s) d s\right) d x d t
\end{aligned}
$$




$$
\begin{array}{r}
=\int_{Q}\left(a\left(\cdot, T_{k}\left(u_{n}\right), \nabla T_{k}\left(u_{n}\right)\right)-a\left(\cdot, T_{k}\left(u_{n}\right), \nabla T_{k}\left(v_{j}\right) \chi_{j}^{s}\right)\right)\left(\nabla T_{k}^{*}\left(u_{n}\right)-\nabla T_{k}\left(v_{j}\right) \chi_{j}^{s}\right) \\
\times \exp \left(\int_{0}^{u_{n}} g(s) d s\right) d x d t+\varepsilon(n, j, s) .
\end{array}
$$

Then

$$
\begin{aligned}
& \int_{Q} a\left(\cdot, T_{k}\left(u_{n}\right), \nabla T_{k}\left(u_{n}\right)\right) \nabla T_{k}^{*}\left(u_{n}\right) d x d t \\
& \leq \int_{Q} a\left(\cdot, T_{k}\left(u_{n}\right), \nabla T_{k}\left(u_{n}\right)\right) \nabla T_{k}^{*}(u) \chi^{s} d x d t \\
& \quad+\int_{Q} a\left(\cdot, T_{k}\left(u_{n}\right), \nabla T_{k}^{*}(u) \chi^{s}\right)\left(\nabla T_{k}^{*}\left(u_{n}\right)-T_{k}(u) \chi^{s}\right) d x d t \\
& +\varepsilon(n, j, \mu, i, s, m) .
\end{aligned}
$$

We deduce that

$$
\begin{aligned}
& \underset{n}{\limsup } \int_{Q} a\left(\cdot, T_{k}\left(u_{n}\right), \nabla T_{k}\left(u_{n}\right)\right) \nabla T_{k}^{*}\left(u_{n}\right) d x d t \\
& \quad \leq \int_{Q} a\left(\cdot, T_{k}(u), \nabla T_{k}(u)\right) \nabla T_{k}^{*}(u) \chi^{s} d x d t+\lim _{n} \varepsilon(n, j, \mu, i, s, m),
\end{aligned}
$$

so that

$$
\begin{aligned}
\underset{n}{\limsup } \int_{Q} a\left(\cdot, T_{k}\left(u_{n}\right),\right. & \left.\nabla T_{k}\left(u_{n}\right)\right) \nabla T_{k}^{*}\left(u_{n}\right) d x d t \\
& \leq \int_{Q} a\left(\cdot, T_{k}(u), \nabla T_{k}(u)\right) \nabla T_{k}^{*}(u) \chi^{s} d x d t \\
& \leq \liminf _{n} \int_{Q} a\left(\cdot, T_{k}\left(u_{n}\right), \nabla T_{k}\left(u_{n}\right)\right) \nabla T_{k}^{*}\left(u_{n}\right) d x d t
\end{aligned}
$$

as $n \rightarrow \infty$. Hence

$$
a\left(\cdot, T_{k}\left(u_{n}\right), \nabla T_{k}\left(u_{n}\right)\right) \nabla T_{k}^{*}\left(u_{n}\right) \rightarrow a\left(\cdot, T_{k}(u), \nabla T_{k}(u)\right) \nabla T_{k}^{*}(u) \text { in } L^{1}(Q) .
$$

Using the same argument as above, we obtain

$$
a\left(\cdot, T_{k}\left(u_{n}\right), \nabla T_{k}\left(u_{n}\right)\right) \nabla T_{k}\left(u_{n}\right) \rightarrow a\left(\cdot, T_{k}(u), \nabla T_{k}(u)\right) \nabla T_{k}(u) \text { in } L^{1}(Q),
$$

and Vitali's theorem and 1.2 give

$\nabla T_{k}\left(u_{n}\right) \rightarrow \nabla T_{k}(u) \quad$ for the modular convergence in $\left(L_{M}(Q)\right)^{N}$.

STEP 4: Passing to the limit. Using the approximate function of Lemma 3.2 of [KMS, the passing to the limit is easy, as in [EM2, EM3].

REMARK 2.3. A similar result can be proved when dealing with the right-hand side in $L^{1}(Q)+W^{-1, x} E_{\bar{M}}(Q)$. 
Acknowledgements. The authors are grateful to Professor Driss Meskine for his comments and suggestions. His article [KMS] was the motivation for writing this article.

\section{References}

[ABT] L. Aharouch, J. Bennouna and A. Touzani, Existence of renormalized solution of some elliptic problems in Orlicz spaces, Rev. Mat. Complut. 22 (2009), 91-110.

[ABM15] Y. Akdim, A. Benkirane and M. El Moumni, Solutions of nonlinear elliptic problems with lower order terms, Ann. Funct. Anal., to appear.

[ABM] Y. Akdim, A. Benkirane and M. El Moumni, Existence results for nonlinear elliptic problems with lower order terms, Int. J. Evolution Equations 8 (2014), 20 pp.

[ABMR] Y. Akdim, A. Benkirane, M. El Moumni and H. Redwane, Existence of renormalized solutions for nonlinear parabolic equations, J. Partial Differential Equations 27 (2014), 28-49.

[AB] E. Azroul and A. Benkirane, On a necessary condition in the calculus of variations in Orlicz-Sobolev spaces, Math. Slovaca 51 (2001), 93-105.

[BE] A. Benkirane and A. Elmahi, Almost everywhere convergence of the gradients of solutions to elliptic equations in Orlicz spaces and application, Nonlinear Anal. 11 (1997), 1769-1784.

[D] T. Donaldson, Inhomogeneous Orlicz-Sobolev spaces and nonlinear parabolic initial-boundary value problems, J. Differential Equations 16 (1974), 201-256.

[E] A. Elmahi, Strongly nonlinear parabolic initial-boundary value problems in Orlicz spaces, Electron. J. Differential Equations Conf. 9 (2002), 203-220.

[EM1] A. Elmahi and D. Meskine, Strongly nonlinear parabolic equations with natural growth terms in Orlicz spaces, Nonlinear Anal. 60 (2005), 1-35.

[EM2] A. Elmahi and D. Meskine, Parabolic equations in Orlicz spaces, J. London Math. Soc. 72 (2005), 410-428.

[EM3] A. Elmahi and D. Meskine, Strongly nonlinear parabolic equations with natural growth terms and $L^{1}$ data in Orlicz spaces, Portugal. Math. 62 (2005), 143-183.

[GM] J.-P. Gossez and V. Mustonen, Variational inequality in Orlicz-Sobolev spaces, Nonlinear Anal. 11 (1987), 379-392.

[KMS] M. Kbiri Alaoui, D. Meskine and A. Souissi, On some class of nonlinear parabolic inequalities in Orlicz spaces, Nonlinear Anal. 74 (2011), 5863-5875.

[L] R. Landes, On the existence of weak solutions for quasilinear parabolic initialboundary value problems, Proc. Roy. Soc. Edinburgh Sect. A 89 (1981), 137217.

[P] A. Porretta, Existence results for nonlinear parabolic equations via strong convergence of truncations, Ann. Mat. Pura Appl. (4) 177 (1999), 143-172.

[R] J. Robert, Inéquations variationnelles paraboliques fortement non linéaires, J. Math. Pures Appl. 53 (1974), 299-321.

[YBM1] A. Youssfi, A. Benkirane and M. El Moumni, Existence result for strongly nonlinear elliptic unilateral problems with $L^{1}$-data, Complex Var. Elliptic Equations 59 (2014), 447-461. 
[YBM2] A. Youssfi, A. Benkirane and M. El Moumni, Bounded solutions of unilateral problems for strongly nonlinear equations in Orlicz spaces, Electron. J. Qualit. Theory Differential Equations 2013, no. 21, 25 pp.

Azeddine Aissaoui Fqayeh, Abdelmoujib Benkirane, Mostafa El Moumni

Laboratory LAMA

Department of Mathematics

Faculty of Sciences Dhar El Mahraz

University Sidi Mohamed Ben Abdellah

P.O. Box 1796 Atlas

Fez, Morocco

E-mail: faqueh.fassi@gmail.com

abd.benkirane@gmail.com

mostafaelmoumni@gmail.com

Received on 25.2.2014;

revised version on 18.7.2014 
